\title{
Epidemiologia da Sífilis Congênita no estado de Sergipe
}

\author{
Epidemiology of Congenital Syphilis in the state of Sergipe \\ Epidemiología de la Sífilis Congénita en el estado de Sergipe
}

\author{
Kirlly Bezerra da Silveira \\ ORCID: https://orcid.org/0000-0002-2136-0246 \\ Universidade Federal de Sergipe, Brasil \\ E-mail: kirlly13@yahoo.com \\ José Rodrigo Santos Silva \\ ORCID: https://orcid.org/0000-0002-1918-7122 \\ Universidade Federal de Sergipe, Brasil \\ E-mail: rodrigo.ufs@gmail.com \\ Francisco Prado Reis \\ ORCID: https://orcid.org/0000-0002-7776-1831 \\ Universidade Tiradentes, Brasil \\ E-mail: franciscopradoreis@gmail.com \\ Ítalo Fernando Lisboa de Melo \\ ORCID: https://orcid.org/0000-0002-1754-8561 \\ Universidade Federal de Sergipe, Brasil \\ E-mail: ítalo-lisboa@hotmail.com \\ Tamires Carvalho de Mendonça Santos \\ ORCID: https://orcid.org/0000-0002-6638-1323 \\ Universidade Federal de Sergipe, Brasil \\ E-mail: thamirescarvalho_1955@hotmail.com \\ Marília de Jesus Souza \\ ORCID: https://orcid.org/0000-0003-3878-7614 \\ Universidade Federal de Sergipe, Brasil \\ E-mail: lilasouza28@outlook.com \\ Vera Lúcia Corrêa Feitosa \\ ORCID: https://orcid.org/0000-0001-5705-6433 \\ Universidade Federal de Sergipe, Brasil \\ E-mail: vera_feitosa@uol.com.br
}

\begin{abstract}
Resumo
Objetivo: O objetivo desse trabalho é analisar a dinâmica temporal da sífilis gestacional e congênita em Sergipe, e descrever características associadas a variáveis sociodemográficas das mães cujos filhos tiveram sífilis congênita em Sergipe no período de 2007 a 2015. Metodologia: foi realizado um estudo descritivo, ecológico, analítico e do tipo série histórica de todos os casos de sífilis congênita ocorridos em Sergipe no ano de 2007 a 2015, incluindo as mães notificadas no Sistema de Informação de Agravos de Notificação - SINAN. Resultados: houve um aumento da taxa de incidência de sífilis congênita no período de 2007 a 2010, e uma tendência de estabilização da doença a partir deste ano a maioria das mães avaliadas, $68,46 \%$ encontra-se entre 20 a 34 anos, $40,7 \%$ dessas mulheres possuem ensino fundamental incompleto e $85 \%$ se declararam pardas, $72,7 \%$ realizaram o pré-natal e mesmo assim boa parte dessas mulheres foi diagnosticada tardiamente. Conclusão: este fato reflete a fragilidade da assistência de pré-natal prestada a estas mulheres no citado estado. Este fato reflete a necessidade de melhorias nas ações dos serviços de saúde em Sergipe. Palavras-chave: Sífilis congênita; Epidemiologia; Sergipe.
\end{abstract}

\begin{abstract}
Objective: The objective of this study is to analyze the temporal dynamics of congenital and gestational syphilis in Sergipe and to describe associated characteristics to sociodemographic variables of mothers whose children had congenital syphilis in Sergipe no from 2007 to 2015. Methodology: a descriptive, ecological, analytical and historical series study of all cases of congenital syphilis occurred in Sergipe in 2007 to 2015, including the mothers notified in the Notification System - SINAN. Results: there was an increase in the incidence rate of congenital syphilis in the period from 2007 to 2010, and a trend towards stabilization of the disease from this year onwards. Most of the mothers evaluated, $68.46 \%$ are between 20 and 34 years old, $40.7 \%$ of these women had incomplete elementary education and $85 \%$ declared themselves to be brown, $72.7 \%$ performed prenatal care and even though a good part of these women were diagnosed late. Conclusion: this fact reflects the fragility of prenatal care provided to these women in the aforementioned state. This fact reflects the need for improvements in the actions of health services in Sergipe.
\end{abstract}

Keywords: Congenital syphilis; Epidemiology; Sergipe. 


\section{Resumen}

Objetivo: El objetivo de este trabajo es analizar la dinámica temporal de la gestación y sífilis congénita en Sergipe, y describen características asociadas a variables sociodemográficas de madres cuyos hijos tuvieron sífilis congénita en Sergipe de 2007 a 2015. Metodología: se realizó un estudio descriptivo, ecológico, analítico e histórico de serie de todos los casos de sífilis congénita ocurridos en Sergipe en 2007 a 2015, incluyendo madres notificadas en el Sistema de Información de Enfermedades Notificables - SINAN. Resultados: hubo un aumento en la tasa de incidencia de sífilis congénita en el período de 2007 a 2010, y una tendencia a la estabilización de la enfermedad a partir de este año, la mayoría de las madres evaluadas, 68,46\% tienen entre 20 y 34 años, 40,7\% de estas las mujeres tienen educación primaria incompleta y el $85 \%$ se declararon morenas, el $72,7 \%$ tuvo atención prenatal y aun así una buena parte de estas mujeres fueron diagnosticadas tardiamente. Conclusión: este hecho refleja la fragilidad de la atención prenatal brindada a estas mujeres en el citado estado. Este hecho refleja la necesidad de mejoras en la actuación de los servicios sanitarios en Sergipe.

Palabras clave: Sífilis congénita; Epidemiología; Sergipe.

\section{Introdução}

A sífilis, até o presente, como em vários outros países, permanece no Brasil como uma doença negligenciada. Em sua forma gestacional tem sido estimado que dois milhões de gestantes sejam infectadas a cada ano no mundo, pela falta de adequado diagnóstico e tratamento, permitindo, desse modo a doença evoluir para quadros mais graves. Isso ocorre principalmente no Sul da África, em que aproximadamente $63 \%$ das gestantes são infectadas com sífilis. Este fato aumentou o interesse das autoridades mundiais em saúde pelo controle da doença, porque $70 \%$ das gestantes infectadas não eram tratadas ou o tratamento aconteceu de forma inadequada transmitindo infecção ao feto, resultando em efeitos adversos como: prematuridade, baixo peso fetal, más formações congênitas, retardo no desenvolvimento da criança além de muitos outros sintomas (Adeyinka et al., 2018; Galvao et al., 2013; Gomez et al., 2013).

No mundo, de acordo com a Organização Mundial de Saúde (OMS), a cada ano, aproximadamente meio milhão de crianças nascem a cada ano com sífilis congênita (SC), enquanto outro meio milhão, são de natimortos e abortos causados pela presença da doença. No Brasil, cerca de 12 mil recém-nascidos contraem sífilis a cada ano. Estes números evidenciam que a doença é um problema de saúde pública o que vem reforçar o compromisso internacional com sua eliminação. Entre as dificuldades de controle da doença têm sido destacados: o diagnóstico tardio; o foco na saúde da mulher; a realização mais adequada do pré-natal; prevenir o risco de reinfecção realizando o tratamento dos parceiros. Dessa maneira espera-se que venha a ser quebrada a cadeia de transmissão da sífilis (Freitas et al., 2021; Hermann et al., 2016; MS, 2015; Taylor et al., 2017).

Como consequência dos elevados números de casos da sífilis, em especial, nos países da América, Ásia-Pacífico e África, a OMS lançou em 2007 uma iniciativa global para eliminar a SC nestas três regiões. Desde então, nesses continentes, com o propósito de melhorar a saúde materna e infantil e contribuir para a realização dos objetivos do milênio, teve início uma serie de esforços locais, que foram centrados na dupla eliminação da transmissão: materno fetal do HIV e da sífilis. Para esse fim, alguns critérios foram estabelecidos objetivando a aceitação dos países para alcançarem as metas do milênio: como: 95\% das mulheres grávidas realizarem os pré-natais; executar em 95\% das mulheres grávidas testes de HIV e sífilis na gravidez; e 95\% das gestantes diagnosticadas com HIV ou sífilis para receberem tratamento. Para transmissão fetal as metas a serem alcançadas são: o HIV , $\leq 50$ de novas infecções pediátricas por 100.000 nascidos vivos e uma taxa de transmissão de $<5 \%$ nas mulheres que amamentam e para sífilis, $\leq 50$ casos de SC por 100.000 nascidos vivos (CONITEC, 2015; Freitas et al., 2021; Taylor et al., 2017).

Nos Estados Unidos, ao contrário de anos anteriores, no ano de 2018, ocorreu um pico da SC que atingiu crianças cujas mães realizaram regularmente as consultas de pré-natal. Na época foi admitida a indicação de que havia a necessidade de uma melhor abordagem nas ações educativas e preventivas da saúde dessas gestantes. Abordagens atuais sugerem que as testagens em relação a doença sejam realizadas no primeiro e no terceiro trimestres da gestação (Rac et al., 2020).

Um estudo em que foram avaliados os sistemas atuais de vigilância epidemiológica da sífilis materna e congênita de 81 
países, por meio de exames de indicadores de cobertura dos serviços de assistências prestadas às gestantes, com a meta de a eliminação da sífilis congênita até 2030 foram identificadas lacunas na assistência do pré-natal. É possível que esta situação possa se refletir no aumento do número de casos associados a SC bem como o aumento de desfechos desfavoráveis na gestação como o aumento dos natimortos(Trivedi et al., 2020).

Embora a SC em Sergipe seja considerada como prioridade nos Programas de Vigilância Epidemiológica, até o presente, a realização de mais estudos, que tratem especificamente sobre o tema, tornam-se necessário. Há necessidade de conhecimento quanto à incidência nas cidades do estado de Sergipe, acompanhada de dados como: a situação sociodemográfica materna, as consultas durante o pré-natal, realização de teste rápido para sífilis e HIV, tratamento dos parceiros, tratamento e estadiamento da doença e sua evolução.

O presente estudo teve por objetivo analisar a dinâmica temporal da sífilis congênita no estado de Sergipe, e descrever a associação de variáveis sociodemográficas referentes às mães e de seus filhos nascidos e diagnosticados como portadores de $\mathrm{SC}$.

\section{Metodologia}

Trata-se de um estudo ecológico misto, utilizando técnicas de análise temporal e espacial, com a utilização de dados secundários das notificações de sífilis congênita (SC) em Sergipe, entre 2007 a 2015. As unidades de análise foram os 75 municípios do estado.

A definição de SC baseou-se nos seguintes critérios: 1) qualquer filho, aborto ou natimorto de mulher com evidência clínica ou sorológica para sífilis, que não tenha sido tratada ou tenha recebido tratamento inadequado; 2) menores de 13 anos com evidência sorológica para sífilis, uma vez que foram excluídas as chances de manutenção de anticorpos maternos e sífilis adquirida; 3) menores de 13 anos com sorologia não treponêmica para sífilis e evidências clínicas, radiológicas ou no líquido cefalorraquidiano (LCR) para SC; ou 4) evidência microbiológica de T. pallidum na placenta, cordão umbilical ou tecido infantil, de aborto ou natimorto (MS, 2015).

Os dados eram de domínio público e abrangiam todos os casos novos confirmados de SC, considerando a $10^{\mathrm{a}}$ revisão da Classificação Internacional de Doenças (CID-10). Os indicadores epidemiológicos foram descritos com taxas de incidência anuais, a partir da razão entre o número de casos novos de SC e os nascidos vivos, residentes no mesmo local e período considerado, e multiplicada pelos constantes 1.000. Os dados foram obtidos diretamente junto ao Sistema de Informação de Agravos de Notificação (SINAN) e ao Sistema de Informação de Nascidos Vivos (SINASC) de Sergipe. Foram excluídos do estudo os casos que apresentavam erros diagnósticos, duplicação, inconsistência ou incompletude nos prontuários. Dados populacionais e grades cartográficas foram obtidos do IBGE (Síntese Do Estado de Sergipe Em 2015, 2015).

Para se estabelecer um paralelo entre a sífilis gestacional e congênita, foi necessário a realização do cálculo de incidência de ambas (sífilis gestacional e congênita) de todos os municípios de Sergipe entre 2007 a 2015 . A partir dos dados obtidos, foi realizada uma análise comparativa entre as duas e verificado até que ponto os bancos de dados com informações das duas doenças (sífilis gestacional e congênita), foram convergentes ou divergentes entre si.

A caracterização da amostra foi realizada por meio de análise descritiva, em que foram calculados as frequências e os percentuais de ocorrência de cada categoria das variáveis qualitativas nominais e ordinais. Foi utilizado o banco de dados de SC para realização das análises estatísticas, e, para uma análise mais apurada foram retirados destas análises todos os casos notificados de SC das mulheres procedentes de outros estados, apesar do parto ter sido realizado em maternidades do estado de Sergipe.

A variável pré-natal foi escolhida, para a realização de cruzamento entre as variáveis sociodemográficas da mãe, como: idade, escolaridade, realização do pré-natal, tratamento desta mulher e de seu parceiro sexual e, nessa segunda fase da pesquisa 
foi realizada uma análise inferencial, realizando o cruzamento entre algumas variáveis, como: idade, ocupação, escolaridade e realização de tratamento. Foram utilizados os testes de Qui-Quadrado de Pearson (Pearson, 1900) e de Exato Fisher (Fisher, 1922).

Em todos os cruzamentos também foi calculada a Razão de Chance (OR), para medir a força das associações entre as variáveis com intervalo de confiança de $95 \%$. O nível de significância adotado foi de 5\%, e as análises foram realizadas no software R 3.5.0. Os casos ignorados duplicados em branco ou com variáveis não preenchidas no banco de dados não foram contados nas análises estatísticas.

O projeto foi aprovado pelo CEP - Comitê de Ética em Pesquisa envolvendo Seres Humanos da Universidade Federal de Sergipe, através do protocolo número: CAAE: 65892316.6.0000.5546.

\section{Resultados}

No período estudado, 01 de janeiro de 2007 a 31 de dezembro 2015, foram notificados em Sergipe, no SINAN, 2.290 casos de sífilis gestacional e 2.381 casos de SC. A diferença entre os dois tipos de sífilis, ou seja, de 91 casos entre os dois tipos de sífilis possivelmente deveu-se à subnotificação durante a gestação das mães.

A taxa de detecção da sífilis gestacional variou de 4,6 em 2007 para 10,7 casos, em 2015, para cada mil nascidos vivos, com incidência média de 7,3 casos por mil nascidos vivos. Enquanto isso a taxa de detecção da SC sífilis congênita de 2,7 em 2007 para 11 em 2015 casos para cada mil nascimentos com taxa de detecção média de 7.5 casos por mil nascidos vivos entre 2007 e 2015, como mostra a Figura 1.

Figura 1: Apresentação estatística de casos de SC em um intervalo de tempo de janeiro de 2007 a dezembro de 2015.

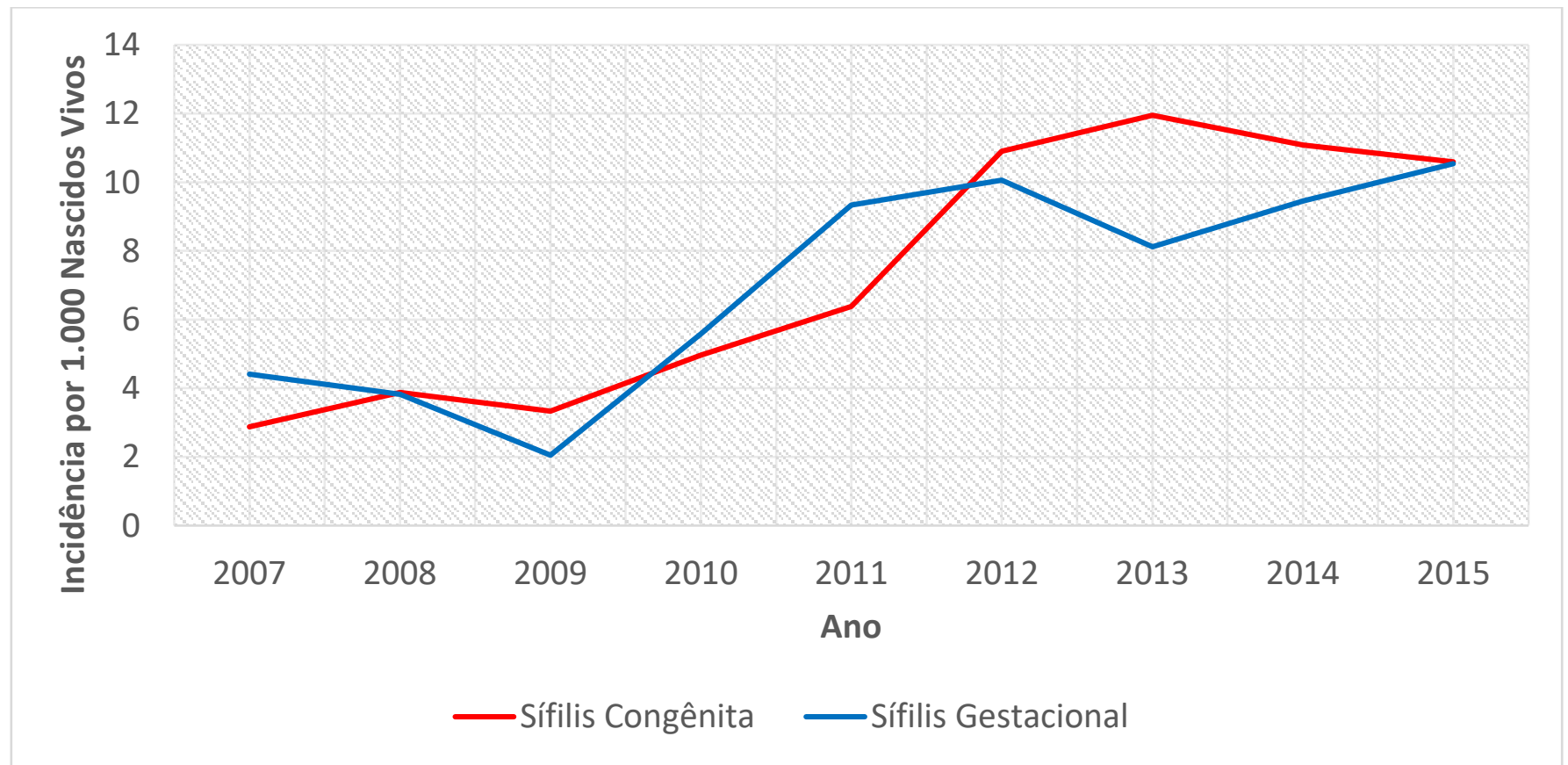

Fonte: Gráfico elaborado pelos autores com dados governamentais do TABNET do Ministério da Saúde. <http://tabnet.datasus.gov.br/cgi/menu_tabnet_php.htm\#>

Na Figura 1, pode ser observado que entre os anos de 2012 a 2015 ocorreu uma maior incidência dos casos de SC comparado aos de sífilis gestacional. Isso possivelmente pode ter ocorrido devido a não realização do diagnóstico da sífilis na 
gestante. Dessa maneira é possível inferir que as ações preconizadas pelo Ministério da Saúde, podem não estarem sendo ofertadas ou realizadas de forma adequada no estado de Sergipe.

A partir do ano de 2011, ocorreu um aumento considerável do número de casos notificados que refletiu sobremodo, também no aumento da incidência dos casos de sífilis gestacional e congênita. Esta ocorrência pode ser creditada às melhorias nos processos de notificação a partir da criação da Rede cegonha, naquele ano e a disponibilização dos testes rápidos nas Unidades de Saúde do SUS (Epidemiológica, 2012).

A tabela 1 demonstra que, embora a grande maioria das mulheres tinha sífilis e transmitiram a doença para seus filhos tenham realizado o pré-natal, elas não tiveram acesso ao diagnóstico da doença em tempo hábil para que fosse instituído o tratamento eficaz. Foi constatado que 43,56\% só tiveram o diagnóstico da doença no momento do parto ou no pós-parto. Este dado pode indicar uma possível fragilidade na qualidade da assistência do pré-natal prestado a essas mulheres, demonstrando de que a realização do pré-natal pode não garantir que as ações preventivas preconizadas estejam sendo realizadas em sua totalidade, ou seja, não garantiu uma boa qualidade de prestação da assistência pré-natal prestada a estas mulheres. Estratégias para detecção precoce e adoção de medidas de tratamento a essas mulheres devem ser fortalecidas e adotadas para buscar uma eficiente assistência o fortalecimento assistencial e de quebrar a cadeia de transmissão da doença da mãe para o feto. Estes resultados ainda sugerem ocorrer falhas determinantes no nível de atenção primária a saúde.

Tabela 1 - Distribuição das mães portadoras de sífilis segundo dados sociodemográficos no estado de Sergipe no período de 2007 a 2015.

\begin{tabular}{|c|c|c|c|}
\hline Variável & Categoria & Frequência & Percentual \\
\hline \multirow[t]{2}{*}{ Realizou Pré-natal ${ }^{(1)}$} & Sim & 1722 & 75.9 \\
\hline & Não & 546 & 24.1 \\
\hline Total & & 2268 & 100 \\
\hline \multirow{4}{*}{ Diagnóstico de sífilis ${ }^{(2)}$ materna } & Durante o pré-natal & 962 & 41,74 \\
\hline & No momento do parto & 1004 & 43,56 \\
\hline & Após o parto & 324 & 14,06 \\
\hline & Não realizado & 15 & 0,65 \\
\hline Total & & 2305 & 100 \\
\hline \multirow[t]{4}{*}{ Faixa Etária da mãe ${ }^{(3)}$} & 10 a 14 & 28 & 1,19 \\
\hline & 15 a 19 & 440 & 18,68 \\
\hline & 20 a 34 & 1614 & 68,54 \\
\hline & 35 a 49 & 273 & 11,59 \\
\hline Total & & 2355 & 100 \\
\hline \multirow[t]{4}{*}{ Escolaridade da Mãe ${ }^{(4)}$} & Analfabeta & 71 & 3,56 \\
\hline & Ensino Fundamental & 1547 & 77,58 \\
\hline & Ensino Médio & 345 & 17,30 \\
\hline & Ensino Superior & 31 & 1,55 \\
\hline Total & & 1994 & 100 \\
\hline \multirow[t]{5}{*}{ Raça/cor da mãe ${ }^{(5)}$} & Branca & 117 & 5,17 \\
\hline & Preta & 123 & 5,44 \\
\hline & Amarela & 6 & 0,27 \\
\hline & Parda & 1993 & 88,11 \\
\hline & Indígena & 23 & 1,02 \\
\hline Total & & 2262 & 100 \\
\hline
\end{tabular}

NOTAS: Dos 2381 casos notificados de Sífilis Congênita em Sergipe:

(1) 113 foram registrados no banco de dados sem informação definida para esta variável (ign/branco).

(2) 76 foram registrados no banco de dados sem informação definida para esta variável (ign/branco).

(3) 26 foram registrados no banco de dados sem informação definida para esta variável (branco).

(4) 387 foram registrados no banco de dados sem informação definida para esta variável (ign/branco). (ign/branco, não se aplica).

(5) 119 foram registrados no banco de dados sem informação definida para esta variável (ign/branco).

Fonte: Dados elaborados pelos autores com dados governamentais. Estatística (2021). 
A Tabela 2 mostra que não houve correlação significativa entre a não realização do pré-natal e a faixa etária dessas mulheres (p-valor=0,275). Por outro lado, existiu uma correlação significativa entre a realização do pré-natal e a ocupação da mãe, $(\mathrm{p}$-valor $=0,0000)$. O percentual para as mães que exerciam uma atividade remunerada foi maior $(85,75 \%)$; entre as mães que eram estudantes $(78,63 \%)$, para o grupo de mães que eram donas de casa e as desempregadas, os percentuais foram de $73,72 \%$ e 57,63\%, respectivamente. Ainda com relação à ocupação, a OR de uma mulher desempregada é 4,42 vezes a de não realizar o pré-natal em comparação com as mães que exercem alguma atividade remunerada.-As mães donas de casa tiveram uma chance 2,15 vezes maior de não realizar o pré-natal em comparação com aquelas que exerciam atividade remunerada. Para as estudantes, o OR estimado não foi significativo uma vez que o valor 1 está dentro do intervalo de confiança $(0,97 ; 2,75)$, ou seja, ao nível de significância de 5\% não há diferença na chance de realização do pré-natal entre mães que são estudantes e aquelas que exercem atividade remunerada.

Tabela 2 - Análise estatística do cruzamento de informações do pré-natal com dados sociodemográficos das mulheres com sífilis no estado de Sergipe entre os anos de 2007 a 2015.

\begin{tabular}{|c|c|c|c|c|c|}
\hline \multirow{2}{*}{ Variável } & \multirow{2}{*}{ Categoria } & \multicolumn{2}{|c|}{ Realizou pré-natal } & \multirow{2}{*}{ OR } & \multirow{2}{*}{$\begin{array}{c}\text { P- } \\
\text { valor }\end{array}$} \\
\hline & & Não & Sim & & \\
\hline \multirow[t]{4}{*}{ Faixa Etária da mãe } & 10 a 14 & $7(25,93)$ & $20(74,07)$ & $1,14(0,46 ; 2,83)$ & 0,2775 \\
\hline & 15 a 19 & $86(20,53)$ & $333(79,47)$ & $0,84(0,58 ; 1,23)$ & \\
\hline & 20 a 34 & $389(25,08)$ & $1162(74,92)$ & $1,09(0,80 ; 1,49)$ & \\
\hline & 35 a 49 & $60(23,44)$ & $196(76,56)$ & 1,00 & \\
\hline \multirow[t]{4}{*}{ Ocupação } & Desempregado & $25(42,37)$ & $34(57,63)$ & $4,42(2,46 ; 7,94)$ & 0,0000 \\
\hline & Dona de Casa & $359(26,28)$ & $1007(73,72)$ & $2,15(1,59 ; 2,90)$ & \\
\hline & Estudante & $25(21,37)$ & $92(78,63)$ & $1,64(0,97 ; 2,75)$ & \\
\hline & Atividade Remunerada & $59(14,25)$ & $355(85,75)$ & 1,00 & \\
\hline \multirow[t]{3}{*}{ Tratamento materno } & Inadequado & $349(23,76)$ & $1120(76,24)$ & $8,77(4,09 ; 18,81)$ & 0,0000 \\
\hline & Não realizado & $180(37,04)$ & $306(62,96)$ & $16,55(7,62 ; 35,97)$ & \\
\hline & Adequado & $7(3,43)$ & $197(96,57)$ & 1,00 & \\
\hline \multirow[t]{2}{*}{$\begin{array}{l}\text { Parceiro (s) } \\
\text { tratado(s) } \\
\text { concomitantemente }\end{array}$} & Não & $499(29,28)$ & $1205(70,72)$ & $6,32(4,06 ; 9,86)$ & 0,0000 \\
\hline & Sim & $22(6,15)$ & $336(93,85)$ & 1,00 & \\
\hline \multirow[t]{5}{*}{ Evolução do caso } & Óbito por outras causas & $3(25,00)$ & $9(75,00)$ & $1,45(0,39 ; 5,40)$ & 0,0000 \\
\hline & Óbito por sífilis congênita & $19(37,25)$ & $32(62,75)$ & $2,59(1,45 ; 4,62)$ & \\
\hline & Aborto & $115(84,56)$ & $21(15,44)$ & $23,88(14,79 ; 38,56)$ & \\
\hline & Natimorto & $48(48,98)$ & $50(51,02)$ & $4,19(2,77 ; 6,32)$ & \\
\hline & Vivo & $360(18,65)$ & $1570(81,35)$ & 1,00 & \\
\hline
\end{tabular}

Fonte: Dados elaborados pelos autores com dados governamentais. Estatística (2021).

Houve correlação significativa entre a não realização do pré-natal com o tratamento das mulheres e de forma concomitante com o tratamento de seu parceiro (p-valor=0,0000). Merece destacar que naqueles casos em que não foi realizado o tratamento do parceiro, 29,28\% deles ocorreram entre parceiros de mulheres que não realizaram o pré-natal. Já os casos em que foi realizado o tratamento concomitante dos parceiros, apenas 6,15\% referem-se a casos em que dentre as mulheres que não realizaram o pré-natal. Através da razão de (OR) foi observado que, a não realização do pré-natal aumenta em 8,77 vezes as chances da mulher realizar o tratamento inadequado e em 16,55 vezes as chances de não realizar o tratamento, no caso do 
tratamento do seu parceiro, a não realização do pré-natal, por parte da gestante aumentou em 6,32 vezes a chance de que o tratamento do parceiro não tenha sido realizado.

Ocorreu correlação significativa entre a não realização do pré-natal e a evolução ou desfecho da gravidez (p-valor = 0,0000). Isso ficou evidente pelos $25 \%$ dos óbitos neonatais por diferentes causas devido à SC ocorrida entre mulheres que não realizaram o pré-natal. Dessa maneira foram registrados: $37 \%$ de óbitos neonatais; $84 \%$ de abortos; e $18 \%$ dos fetos nasceram vivos. Através da razão do (OR) foi constatado que, a não realização do pré-natal aumentou em 2,59 vezes as chances da mulher ter um filho que tenha óbito por SC, 4,19 vezes a possibilidade da criança nascer morta em 23,88 vezes a probabilidade de abortamento.

\section{Discussão}

De acordo com os presentes achados pode-se inferir que embora seja totalmente evitável, a SC ainda é um grave problema de saúde pública em Sergipe. A análise dos dados do presente estudo constatou ocorrer no estado de Sergipe, uma divergência: o número de casos notificados de SC foi maior que os da sífilis gestacional.

A análise das séries temporais em Sergipe evidenciou que houve uma tendência crescente tanto da sífilis gestacional quanto da congênita. A SC, no estado apresentou uma taxa de detecção muito acima da preconizada pelo Ministério da Saúde, que é de 0,5 casos por mil nascidos vivos (Guia da Vigilância em Saúde, 2017).

O estudo realizado por Saab (Saab \& Tomaz, 2016) com o objetivo de conhecer a prevalência, a ocorrência de aborto e a idade de mulheres gravidas com infecção por sífilis em Sergipe concluiu que foi elevado o registro de abortos em gestantes na maior parte dos municípios do estado. As taxas de prevalência e incidência de sífilis foram maiores que as aceitas pela OMS, e que a sífilis pode ser um fator associado à ocorrência de alta incidência de aborto e morbimortalidade perinatal no estado.

Em estudo prospectivo, observacional acerca da adesão e acompanhamento de recém-nascidos de mães com sorologia positivo para o Treponema pallidum, realizado na Maternidade Filantrópica de Aracaju, entre 2010 e 2014 , evidenciou uma incidência média entre os anos estudados de 10,2 casos para cada mil nascidos vivos, $28 \%$ dos recém-nascidos não compareceram à primeira consulta para de tratamento da doença, a taxa de abandono do tratamento foi de 75\%, o intervalo médio de cura desses recém-nascidos foi de 2,25 meses, dessa forma, a alta incidência da sífilis foi associadas à baixa adesão ao tratamento, apenas 67,1\% dos recém-nascidos foram tratados com Penicilina Cristalina (Lopes et al., 2016).

Um estudo realizado em um hospital infantil de Buenos Aires, que analisou 61 casos de crianças diagnosticadas com SC entre 1987 e 2019, verificou que a distribuição desses casos apresentou uma curva bimodal entre os anos de 1992 e 1993 e entre 2014 e 2017 a idade média de diagnósticos casos foi de 2 meses e os principais achados foram: alterações ósseas, hepatoesplenomegalias, anemia, lesões cutâneas e comprometimento renal (Garcia et al., 2021).

Entre os anos 2010 e 2011 ocorreu um número muito elevado de casos de SC, com aumento das notificações de $34 \%$ no Brasil (Rac et al., 2020). No estado de Sergipe, assim como nos demais estados brasileiros as infecções sexualmente transmissíveis (IST), são grave problema de saúde pública em virtude dos indicadores estaduais estarem em consonância com os nacionais o estado de Sergipe adotou ações mais severas e eficazes com o objetivo de controlar a doença. Surgiram algumas ações na busca do controle desse agravo, dentre elas estão ações como a solicitação do VDRL para os homens na rotina dos atendimentos nos serviços de saúde prestados pelo SUS no estado (DCCI/SVS, 2021).

A literatura mostra que a relação da sífilis com fatores sociodemográficos foi distribuída de forma desigual no mundo. Em um estudo realizado na China sobre o perfil epidemiológico das mulheres com sífilis, foi evidenciado um aumento abrupto no número de casos notificados desta doença, passando de 0.1 casos por mil nascidos vivos em 1991 para 69,9 casos por mil nascidos vivos (NV) em 2013 (Dou et al., 2016). No mesmo estudo foram analisadas características sociodemográficas, econômicas, bem como, informações gerais do pré-natal, tais como: número de consultas realizadas, se houve o diagnóstico da 
doença em tempo hábil, se houve tratamento da mãe e do seu parceiro, se este foi realizado de forma correta dentre outras informações para tentar entender as causas do problema. Os resultados mostraram que a maioria das mulheres, $79,1 \%$, recebeu o diagnóstico da doença antes de 37 semanas de gravidez, 55,4\%, não receberam tratamento ou receberam de forma inadequada, $14 \%$ tiveram sérios problemas no desfecho da gravidez, como: abortamento, natimortalidade, baixo peso ao nascer e o aparecimento da SC.

Peterman e colaboradores (2015) estimaram as taxas e tendências da infecção por sífilis em grupos de populações nos Estados Unidos, e relataram que em 2013 as taxas de sífilis primária e secundária, entre os homens que praticavam sexo com homens eram altas, em torno de (228,8 por 100 000) (Peterman et al., 2015). Para as mulheres, em quem a ocorrência desses tipos de sífilis é muito menos comum a taxa foi de (0,9 por 100 000) e ocorria em surtos isolados em alguns municípios com baixos níveis de infecção persistentes. Os autores afirmaram que entre mulheres a tendência da SC seguiria semelhantemente a das sífilis primária e secundária. Essa tendência teria implicações na disseminação da doença. Consideraram que o controle da sífilis através de notificação, triagem e tratamento adequados como ferramentas importantes para o controle dessa infecção sexualmente transmissível.

Lago (2016) realizou estudos relacionado a prevenção e transmissão da sífilis de mãe para filho, enfatizando sobre o diagnóstico e o tratamento da sífilis na gravidez, destacou que, a epidemiologia da sífilis é influenciada por fatores socioeconômicos e culturais, e que os países pobres e em desenvolvimento identificaram a gravidade do problema e que, diante desta realidade enfrentam grandes desafios (Lago, 2016).

A assistência pré-natal é entendida em linha geral como sendo um conjunto de ações e medidas direcionado às mulheres grávidas que incluem: orientações, exames, consultas e o rastreamento da presença de algumas doenças. Essas ações buscam detectar precocemente algumas doenças na mãe e no feto e em especial, com o diagnóstico precoce e tratamento adequado reduzir a incidência da SC e da transmissão do HIV da mãe para o bebê (Rac et al., 2020).

O Ministério da Saúde preconiza que sejam realizadas no mínimo seis consultas de pré-natal ao longo da gestação e que, os testes diagnósticos de HIV e sífilis sejam realizados o mais precocemente e se possível, logo na primeira consulta da gestante ao pré-natal e repetido no terceiro trimestre gestacional. Foi observado que, caso esta orientação tivesse sido executada de maneira satisfatória, possivelmente no estado de Sergipe, não teria ocorrido um número tão elevado de SC (Rac et al., 2020).

Apesar do rastreamento e tratamento da sífilis, ao lado do custo efetivo de realização do pré-natal a presença ainda constante da doença continua a contribuir para o aumento da sua morbimormortalidade em todo o mundo. Adeyinka e colaboradores (2017), em um estudo realizado na Nigéria, avaliaram a prevalência, a tendência de cobertura e o tratamento da sífilis gestacional no programa nacional de prevenção de transmissão vertical da SC e o progresso na eliminação da doença. Estes autores constataram que ao longo dos anos de 2013 a 2016, período de realização do estudo, um aumento do número da realização dos exames sorológicos, destacaram, entretanto, que enquanto a prevalência de sífilis materna tenha diminuído de e 3,2\% em 2013 para 1,4\% em 2016, a cobertura do tratamento da sífilis durante a gravidez diminuiu de 71,3\% em 2013 para $54,9 \%$ em 2016. Por fim concluíram que o rastreamento e o tratamento da sífilis gestacional, foram inadequados para atender as aspirações de eliminação da doença e admitiram que, uma rápida ampliação do rastreamento, tratamento da sífilis e de cuidados pré-natais são cruciais para evitar uma epidemia naquele país até o ano de 2020 (Adeyinka et al., 2018).

No Japão, Kamb e colaboradores (2018) relataram a ocorrência de uma taxa crescente de SC maior nas mulheres japonesas jovens em idade reprodutiva entre de 20 a 29 anos (Saab \& Tomaz, 2016). O Ministério da Saúde, Trabalho e BemEstar japonês reconheceu esses dados como uma ameaça à saúde pública e fortaleceu suas campanhas de prevenção para rastreamento e tratamento da sífilis visando atender, em especial, a este grupo populacional (Kamb et al., 2018).

Na Colômbia, Garcés e colaboradores (2017) em um estudo que teve como objetivo identificar lacunas entre os profissionais de saúde na prevenção da transmissão de sífilis / HIV de mãe para filho e fornece recomendações para apoiar esses 
programas, comprovaram entre os trabalhadores de saúde pesquisados, incluindo médicos a presença de déficits de conhecimento no manejo da sífilis gestacional. Destacaram ainda, que na realização dos testes rápidos para sífilis, utilizados em laboratórios clínicos em Cali, havia deficiências processuais em seu uso, incluindo garantia de controle de qualidade e que os profissionais de saúde estavam interessados em identificar e tratar a sífilis gestacional em um único evento. Estes autores sugeriram a necessidade de treinamento intensivo dirigido a profissionais de saúde e de políticas públicas voltadas para grupos acadêmicos para garantir a implementação adequada de novas estratégias para a prevenção da transmissão de sífilis / HIV de mãe para fillho (Garcés et al., 2017).

O conhecimento dos profissionais da atenção básica sobre a doença, que no caso é sífilis em todas as suas apresentações, adquirida, gestacional e congênita é um fator importante no controle e cura deste agravo. Em estudo transversal realizado por Rodrigues, DC, 2017 entre janeiro e maio de 2015, sobre o conhecimento, manejo e práticas dos profissionais de saúde que trabalham no pré-natal sobre a sífilis durante a gravidez identificou que apenas 2 critérios, "conhecimento sobre a transmissão de mãe a criança de acordo com a idade gestacional" e "aconselhamento sobre infecção", atingiram 95\% de conformidade (Rodrigues \& Domingues, 2018).

De acordo com o mesmo estudo o conhecimento do perfil epidemiológico da SC sífilis congênita e o objetivo de sua da eliminação e o conhecimento sobre testes sorológicos apresentaram escolares em Teresina -PI evidenciou que o conhecimento está abaixo de 50\%, por parte de médicos e enfermeiros enquanto as práticas relacionadas ao aconselhamento pós-teste, ao controle da cura e ao tratamento dos parceiros atingiram 60\%. O estudo mostrou também que barreiras organizacionais relacionadas ao início tardio do pré-natal, o retorno atrasado dos resultados do teste da sífilis, o tratamento adequado da doença com a aplicação da penicilina benzatina nas unidades de atenção primária e ao tratamento dos parceiros são problemas identificados. Resultado semelhante foi identificado em Sergipe, ambos necessitam de ações como: educação continuada direcionada aos profissionais de saúde que atuam no Estratégia Saúde da família, início precoce da pré-natal e tratamento adequado desta gestante e de seu parceiro são ações necessárias para o controle da SC no estado sergipano (Rodrigues \& Domingues, 2018).

Diante do cenário representado por números elevados de pessoas infectadas pela sífilis, justifica-se diante da ausência de orientações necessárias ou até mesmo, a sintomatologia, estágio com apresentações variáveis, longos períodos de latência da infecção e escassa procura nos serviços de atendimentos em saúde. Além disso, a adesão baixa ao pré-natal contribui fortemente para o diagnóstico tardio da Sífilis Gestacional ou Sífilis Congênita. O aumento crescente de novos casos de sífilis na população em geral pode ser atribuído a baixa na cobertura de testagem, não adesão aos preservativos masculinos ou femininos e desabastecimento mundial da Penicilina. Além disso, o aprimoramento do sistema de vigilância ao longo dos anos pode refletir significativamente no número de casos notificados (de Souza Câmara et al., 2021; dos Santos Aleluia et al., 2021; Zaupa et al., 2020).

\section{Conclusão}

Os achados do presente estudo demonstraram a ocorrência de elevados índices de sífilis congênita no estado de Sergipe. Esta ocorrência pareceu estar relacionada, em especial, à fatores sociodemográficos das mulheres infectadas. Por outro lado, foram encontrados indícios de que as ações desenvolvidas durante o pré-natal, podem não estar sendo executadas de maneira efetiva e eficientes. Contudo, sugerimos o fortalecimento das ações desse pré-natal e a implementação eficiente de uma rotina de testagem para detecção da sífilis e do acompanhamento do pré-natal. Sugerimos que mais estudos sejam realizados visando novas intervenções entre as gestantes e puérperas, para melhoria dos indicadores epidemiológicos da sífilis congênita em Sergipe. 


\section{Agradecimentos}

Agradecemos à Secretaria Municipal de Saúde de Aracaju e ao Instituto Brasileiro de Geografia (IBGE) pelo apoio e assessoria durante toda a coleta de dados.

\section{Referências}

Adeyinka, D. A., Olakunde, B. O., Ozigbu, C. E., Agogo, E. A., Morka, M., Atoba, T., Obanubi, C., Okorie, G., Davies, A., \& Oladimeji, O. (2018). Elimination of mother-to-child transmission of syphilis: Is it a reality in Nigeria by 2020? Scandinavian Journal of Public Health, 46(8), 794-797.

CONITEC, C. N. de I. de T. (2015). Penicilina benzatina para prevenção da Sífilis Congênita durante a gravidez. Ministério da Saúde Brasília.

DCCI/SVS, D. de D. de C. C. e I. S. T. (2021). Infecções Sexualmente Transmissíveis.

de Souza Câmara, L., da Silva, L. R., de Oliveira Guerra, B. C., da Costa Monnerat, I., Martins, C. J., Veras, R. C., de Lima Moraes, L. A., da Silva Pinheiro, I., Teixeira, S. V. B., \& Ribeiro, M. S. de F. G. (2021). Conhecimento técnico dos profissionais de saúde quanto ao manejo da sífilis e a sua relação com a Educação Permanente em Saúde. Research, Society and Development, 10(2), e2010211996-e2010211996.

dos Santos Aleluia, E., dos Reis Nascimento, L., da Silva Bomfim, V. V. B., Rodrigues, A. S., Arruda, M. D. I. S., do Nascimento Oliveira, A. R., de Barros Silva, M. E. W., da Silva Couto, S. I., Ramos, T. H. V., \& de Oliveira Holanda, D. (2021). Repercussões da sífilis na gestação: Possibilidades de atuação da enfermagem. Research, Society and Development, 10(7), e51710716944-e51710716944.

Dou, L., Wang, X., Wang, F., Wang, Q., Qiao, Y., Su, M., Jin, X., Qiu, J., Song, L., \& Wang, A. (2016). Epidemic profile of maternal syphilis in China in 2013. BioMed Research International, 2016.

Epidemiológica, V. (2012). Hepatites Virais. Brasília: Ministério Da Saúde.

Síntese do Estado de Sergipe em 2015, (2015).

Fisher, R. A. (1922). On the interpretation of $\chi 2$ from contingency tables, and the calculation of P. Journal of the Royal Statistical Society, 85(1), 87-94.

Freitas, F. L. S., Benzaken, A. S., Passos, M. R. L. de, Coelho, I. C. B., \& Miranda, A. E. (2021). Protocolo Brasileiro para Infecções Sexualmente Transmissíveis 2020: sífilis adquirida. Epidemiologia e Serviços de Saúde, 30.

Galvao, T. F., Silva, M. T., Serruya, S. J., Newman, L. M., Klausner, J. D., Pereira, M. G., \& Fescina, R. (2013). Safety of benzathine penicillin for preventing congenital syphilis: a systematic review. PLoS One, 8(2), e56463.

Garcés, J. P., Rubiano, L. C., Orobio, Y., Castaño, M., Benavides, E., \& Cruz, A. (2017). Educating health workers is key in congenital syphilis elimination in Colombia. Biomédica, 37(3), 416-424.

Garcia, L. N., Destito Solján, A., Moroni, S., Falk, N., Gonzalez, N., Moscatelli, G., Ballering, G., García Bournissen, F., \& Altcheh, J. M. (2021). Congenital syphilis in Argentina: Experience in a pediatric hospital. PLoS Neglected Tropical Diseases, 15(1), e0009010.

Gomez, G. B., Kamb, M. L., Newman, L. M., Mark, J., Broutet, N., \& Hawkes, S. J. (2013). Untreated maternal syphilis and adverse outcomes of pregnancy: a systematic review and meta-analysis. Bulletin of the World Health Organization, 91, 217-226.

Hermann, A., Silva, M. L., Chakora, E. S., \& Lima, D. C. (2016). Guia do pré-natal do parceiro para profissionais de saúde. Ministério Da Saúde.

Kamb, M. L., Taylor, M. M., \& Ishikawa, N. (2018). Rapid increases in syphilis in reproductive-aged women in Japan: a warning for other countries? Sexually Transmitted Diseases, 45(3), 144.

Lago, E. G. (2016). Current perspectives on prevention of mother-to-child transmission of syphilis. Cureus, 8(3).

Lopes, I. M. D., Aragão, J. A., Lopes, A. D., Almeida-Santos, M., Lima, S. O., Fonseca, V., Feitosa, V. L. C., \& Reis, F. P. (2016). Adhesion to the monitoring of newborns from VDRL positive mothers. MedicalExpress, 3.

Guia da Vigilância em Saúde, (2017).

MS, M. da S. do B. (2015). Protocolo clínico e diretrizes terapêuticas para prevenção da transmissão vertical de HIV, sífilis e hepatites virais. Ministério da Saúde Brasília.

Pearson, K. (1900). X. On the criterion that a given system of deviations from the probable in the case of a correlated system of variables is such that it can be reasonably supposed to have arisen from random sampling. The London, Edinburgh, and Dublin Philosophical Magazine and Journal of Science, 50(302), 157175 .

Peterman, T. A., Su, J., Bernstein, K. T., \& Weinstock, H. (2015). Syphilis in the United States: on the rise? Expert Review of Anti-Infective Therapy, 13(2), $161-168$.

Rac, M. W. F., Stafford, I. A., \& Eppes, C. S. (2020). Congenital syphilis: a contemporary update on an ancient disease. Prenatal Diagnosis, 40(13), 1703-1714.

Rodrigues, D. C., \& Domingues, R. M. S. M. (2018). Management of syphilis in pregnancy: Knowledge and practices of health care providers and barriers to the control of disease in Teresina, Brazil. The International Journal of Health Planning and Management, 33(2), 329-344.

Saab, F., \& Tomaz, C. (2016). Prevalencia de sífilis en mujeres gestantes atendidas por el Programa de Protección a la Gestante (PPG) y reporte de aborto en el 
Research, Society and Development, v. 10, n. 14, e562101422061, 2021

(CC BY 4.0) | ISSN 2525-3409 | DOI: http://dx.doi.org/10.33448/rsd-v10i14.22061

Estado de Sergipe, Brasil. Revista de La Universidad Industrial de Santander. Salud, 48(2), 214-220.

Taylor, M. M., Kamb, M., Wu, D., \& Hawkes, S. (2017). Syphilis screening and treatment: integration with HIV services. Bulletin of the World Health Organization, 95(9), 610

Trivedi, S., Taylor, M., Kamb, M. L., \& Chou, D. (2020). Evaluating coverage of maternal syphilis screening and treatment within antenatal care to guide service improvements for prevention of congenital syphilis in Countdown 2030 Countries. Journal of Global Health, 10(1).

Zaupa, A. B. P., dos Santos, E. B., Detogni, A. C., Medina, B. A. L., Ferronatto, G. F., Pezzini, R. P., Naufel, F. S., \& Boleta-Ceranto, D. de C. F. (2020). Sífilis: uma revisão da literatura. Research, Society and Development, 9(10), e3319108255-e3319108255. 